IP Periodica Polytechnica Chemical Engineering

60(3), pp. 201-209, 2016

DOI: $10.3311 /$ PPch. 8808

Creative Commons Attribution (i)

RESEARCH ARTICLE

\section{Multi-Objective Optimization Approach to Enhance Ethylbenzene Dehydrogenation in the Multi-Stage Spherical Reactors}

\author{
Mohammad Farsi ${ }^{1 *}$, Hani Jowkari ${ }^{1}$, Amir Izad Doust ${ }^{1}$ \\ Received 04 September 2015; accepted after revision 24 January 2016
}

\begin{abstract}
This paper focuses on modeling and optimization of multi-stage spherical reactors to produce styrene through ethylbenzene dehydrogenation. A steady state heterogeneous mathematical model is developed based on the mass and energy conservation laws to predict the performance of the proposed configuration. For proving the accuracy of the considered model, simulation results are compared with the available plant data. In optimization stage, the optimal feed temperature is calculated to maximize styrene production and minimize toluene and benzene production based on the multi-objective model and Pareto frontier curve. To achieve a single optimal point, the multiobjective model is converted to a single objective problem by weighted sum method. The simulation results show that styrene production is improved about $60000 \mathrm{kmol}$ per year compared to conventional configuration. It is proved that lower pressure drop is one of main advantages of the proposed configuration.
\end{abstract}

\section{Keywords}

Ethylbenzene dehydrogenation, Spherical reactor, Heterogeneous model, Multi-objective optimization

\footnotetext{
${ }^{1}$ Department of Chemical Engineering, School of Chemical and Petroleum Engineering, Shiraz University, Shiraz, Iran

*Corresponding author, e-mail: farsi@shirazu.ac.ir
}

\section{Introduction}

Currently due to increasing demand for polymers, researchers have focused on the catalytic dehydrogenation of ethylbenzene to produce styrene as one of the most important monomers in the chemical industry. Styrene is a basic raw material to produce various polymers such as polystyrene, acrylonitrile-butadiene-styrene, styrene-butadiene rubber and styrene-acrylonitrile copolymers [1]. Since styrene plant has large capacity, the investment cost of that plant is high, so any small improvement in the process quality and capacity could yield a considerable financial reward. In this process, crude ethylbenzene is mixed with superheated steam and passes over a solid catalyst bed. The most common catalysts in ethylbenzene dehydrogenation process are based on iron oxide promoted by several percent potassium oxide or potassium carbonate [2].

There are some articles in the literature that discuss about ethylbenzene dehydrogenation. Abdalla et al. developed a detailed kinetics model for dehydrogenation of ethylbenzene to styrene on promoted iron oxide catalyst [3]. Herman et al. modeled the catalytic dehydrogenation of ethylbenzene to styrene in a tubular membrane reactor at steady state condition [1]. The simulation results showed that increasing pressure in the membrane reactor increases ethylbenzene conversion to $90 \%$. Clough and Ramirez developed a mathematical model to investigate the performance of a styrene pilot reactor [4]. They found the optimal location of steam injection ports along the catalytic bed to maximize styrene production. Tamsilian et al. derived a kinetic model based on the Hougen-Watson non-porosity formalism for ethylbenzene dehydrogenation and oxidation reactions on $\mathrm{Fe}_{2} \mathrm{O}_{3}$ catalyst [5]. The results showed that the developed catalyst increases ethylbenzene conversion and decreases required feed temperature in the process. Mousavi et al. simulated styrene production in a fixed bed reactor at steady state condition [6]. They developed a three layers perceptron neural network using results of developed mathematical model to simulate the effect of feed composition and operation condition on conversion and selectivity. Elnashaie developed a detailed mathematical model based on the dusty gas model to analyze the operability of the styrene reactor [7]. Abo-Ghander et al. modelled and optimized 
coupling of ethylbenzene dehydrogenation and nitrobenzene hydrogenation reactions in an integrated membrane reactor, where both hydrogen and heat are transferred across the surface of membrane tubes $[8,9]$. The simulation results suggest that coupling the considered reactions in a single reactor could be feasible and beneficial. It was found that styrene yield approaches to $97 \%$ considering styrene production as the objective function, when nitrobenzene conversion reaches to $80 \%$ considering nitrobenzene conversion as the objective based on developed Pareto set. Farsi et al. modelled and optimized styrene production in an industrial hydrogen perm-selective membrane reactor [10]. The results showed that hydrogen removal from reaction zone increases production capacity.

Since in multi-objective optimization problems different and often contrary objectives are simultaneously satisfied, there is no single global solution and it is necessary to find out a set of points that illustrate the optimal trade-off between objectives, i.e. Pareto frontier. All points on the Pareto frontier are mathematically equivalent and there is no choice among different solutions on the Pareto set. Although there are many alternatives on the Pareto set, however an operator needs one solution [11]. One of the suitable approaches to convert a multiobjective optimization problem to a single objective problem is the prior preference procedure. In prior preference method, one can approximate the relative importance of different objective functions by using higher-level information. Based on this estimation, the multi-objective optimization model is transferred to a single objective problem and the new problem is solved by classical or evolutionary programming. The weighted product and sum methods convert a multi-objective problem into a single objective model by multiplication and summation of different objectives considering user-defined power, respectively [3]. In this method, power of objectives is under influence of decision maker's assessment and different solutions are achieved for the same problem based on their preferences. Bhaskar et al. presented a good review about application of multi-objective optimization methods and Pareto set in chemical engineering area over the last twenty years [12].

In industrial plants and particularly chemical reactors, the pressure drop is a serious problem. The radial flow spherical and radial flow tubular packed bed reactors have lower pressure drop compared to conventional axial flow reactors. Requiring lower thickness is another advantage of spherical reactors compared to tubular reactors. Also, the smaller catalytic pellets with higher effectiveness factor and higher feed molar flow rate can be applied because of lower pressure drop in this configuration. In this paper, the styrene production through dehydrogenation of ethylbenzene over commercial potassium-promoted iron catalyst in the multi-stage spherical reactor is modelled. Spherical reactor includes two concentric spherical shells that empty space between two shells is filled with the catalyst pellets. The aim of the present work is modification of styrene synthesis process for a sustainable development in the ethylbenzene conversion and process selectivity considering a multi-objective optimization model. In Section 2 ethylbenzene dehydrogenation in the conventional and proposed configurations is explained. In Sections 3 and 4 the mathematical model of the conventional, proposed configuration and numerical solution method are presented, respectively. To present an exact mathematical model, as well as styrene dehydrogenation reaction, catalytic and thermal side reactions such as ethylbenzene hydrogenation, cracking and styrene hydrogenation and steam reforming reactions are considered in the kinetic model, too. In Section 5, the considered procedure to develop multi-objective function, optimal Pareto set, and converting it to the single objective problem are explained. In Section 6 the simulation results of the conventional and optimized proposed configurations are presented and compared against the base case at steady state condition.

\section{Process description}

The conventional styrene process consists of three adiabatic tubular axial flow reactors. Output stream from the first and second reactors are heated in a furnace and fed to the second and third reactors. Since the reactors are adiabatic and ethylbenzene dehydrogenation is a reversible endothermic reaction, preheaters are placed to heat the feed stream to provide required heat of reaction. The feed is combined with superheated steam to reduce partial pressure of ethylbenzene and decrease coke formation and carbon fouling. The unconverted reactant and produced styrene and side-products are fed to separation section to produce pure styrene. To prevent polymerization of styrene in the distillation tower short residence time, low temperature, and inhibitor agent is necessary. In this research, axial flow reactors in the styrene plant are substituted by three radial flow spherical reactors to achieve a lower pressure drop and increasing styrene production capacity. Figure 1, shows is a schematic depiction of a spherical reactor.

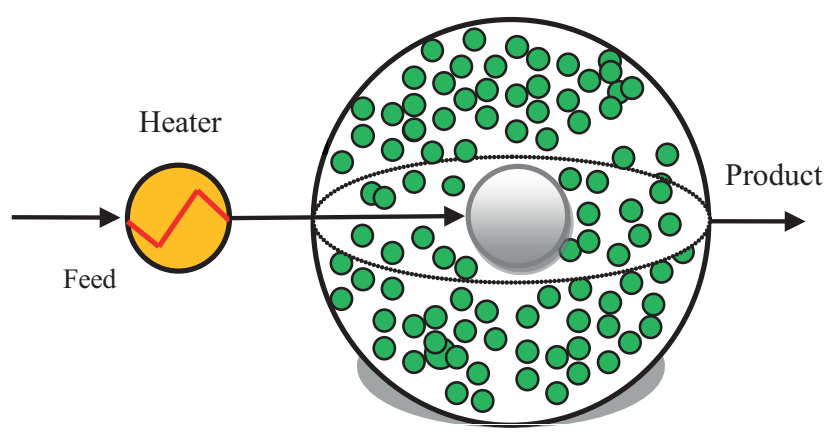

Fig. 1 Schematic depiction of a spherical reactor

The spherical reactor is two concentric perforated sphere vessels that the space between the spheres is filled by catalyst pellets. The proposed configuration includes three reactors that they have been connected in series. Since the net of total mol increases through the reaction, therefore the feed flows form 
inner vessel toward the outer spherical vessel to maintain pressure drop at minimum value.

\section{Process Modelling}

In this research, a one-dimensional heterogeneous model is developed to simulate the conventional and proposed configurations at steady state condition.

\subsection{Axial Flow Reactor}

The mathematical model of axial flow tabular reactor is developed based on the following assumptions:

- The gas mixture is in ideal condition (low pressure and high temperature)

- Radial diffusion of mass and energy is negligible.

- The temperature gradient in the solid phase is negligible.

- The feed flows uniformly in the axial direction of tubular reactors

The mass and energy balance equations for the gas phase in an element of axial flow reactor are as following:

$$
\begin{gathered}
-u_{s} \frac{d C_{i}}{d z}-k_{g i} a_{v}\left(C_{i}-C_{i}^{s}\right)=0 \\
-u_{s} \rho_{g} C_{p} \frac{d T}{d z}-h_{f} a_{v}\left(T_{s}-T\right)=0
\end{gathered}
$$

In heterogeneous models, the concentration and temperature difference between gas phase and solid phase is considered in the developed model. The conservation equations for the solid phase are as:

$$
\begin{gathered}
k_{g} a_{v}\left(C_{i}-C_{i}^{s}-\eta \rho_{B} r_{i}\right)=0 \\
h_{f} a_{v}\left(T_{s}-T\right)-\eta \rho_{B} r(-\Delta H)=0
\end{gathered}
$$

The pressure drop throughout the catalyst bed for spherical reactor is derived based on the Ergun equation as:

$$
\frac{d P}{d z}=\frac{150 \mu(1-\varepsilon)^{2}}{\varphi^{2} d_{p}^{2} \varepsilon^{3}} \frac{Q}{A_{c}}+\frac{1.75 \rho(1-\varepsilon)}{\varphi d_{p} \varepsilon^{3}} \frac{Q^{2}}{A_{c}}
$$

The considered boundary condition of the first axial flow reactor is:

$$
\text { at } \mathrm{x}=0 \quad\left[\begin{array}{c}
P=P_{0} \\
T=T_{0} \\
C=C_{0}
\end{array}\right.
$$

The outlet pressure and components concentration from first and second reactors are the inlet condition of second and third reactors, respectively.

\subsection{Radial Flow Reactor}

The following assumptions are applied in the radial flow spherical reactor:

- The gas mixture is in ideal condition (low pressure and high temperature)

- The temperature gradient in the solid phase is negligible.

- The feed flows uniformly in the radial direction.

The mass and energy balance equations for the gas phase in an element of radial flow spherical reactors are as:

$$
\begin{aligned}
& D_{e r} \frac{1}{r^{2}} \frac{d}{d}\left(r^{2} \frac{d C_{i}}{d r}\right)-\frac{1}{r^{2}} \frac{d}{d}\left(r^{2} u_{r} C_{i}\right)-k_{g} a_{v}\left(C_{i}-C_{i}^{s}\right)=0 \\
& k_{e r} \frac{1}{r^{2}} \frac{d}{d}\left(r^{2} \frac{d T}{d r}\right)-\frac{1}{r^{2}} \frac{d}{d}\left(r^{2} \rho u_{r} C_{p} T\right)-h_{f} a_{v}\left(T_{s}-T\right)=0
\end{aligned}
$$

The conservation equations for the solid phase are as:

$$
\begin{gathered}
k_{g} a_{v}\left(C_{i}-C_{i}^{s}\right)-\eta \rho_{B} r_{i}=0 \\
h_{f} a_{v}\left(T_{s}-T\right)-\eta \rho_{B} r(-\Delta H)=0
\end{gathered}
$$

By neglecting radial mass dispersion and heat conductivity, the conservation equations in the fluid phase are as follows:

$$
\begin{gathered}
-\frac{1}{r^{2}} \frac{d}{d}\left(r^{2} u_{r} C_{i}\right)-k_{g} a_{v}\left(C_{i}-C_{i}^{s}\right)=0 \\
-\frac{1}{r^{2}} \frac{d}{d}\left(r^{2} \rho u_{r} C_{p} T\right)-h_{f} a_{v}\left(T_{s}-T\right)=0
\end{gathered}
$$

It was mentioned that radial flow reactors offer a larger mean cross-sectional area and reduced travel distance as compared to traditional axial flow reactors. The pressure drop through the radial flow reactor is calculated based on the Ergun equation:

$$
\frac{d p}{d z}=\frac{150 \mu(1-\varepsilon)^{2}}{\varphi^{2} d_{v}^{2} \varepsilon^{3}} \frac{Q}{4 \pi}+\left(\frac{1.75 \rho(1-\varepsilon)}{\varphi d_{\nu} \varepsilon^{3}} \frac{Q^{2}}{(4 \pi)^{2}}\right) \frac{1}{r^{4}}
$$

The considered boundary condition of the first radial flow reactor is as:

$$
\text { at } \mathrm{R}=R_{\text {inner }} \quad\left[\begin{array}{c}
P=P_{0} \\
T=T_{0} \\
C=C_{0}
\end{array}\right.
$$

The outlet pressure and components concentration from first and second reactors are the inlet condition of second and third reactors, respectively. Table 1 represents the catalyst, feed specifications, and design data of industrial adiabatic reactors. 


\subsection{Kinetic model}

Ethylbenzene dehydrogenation is a reversible endothermic reaction associated with an increase in number of moles. The reactant is mixed with steam and reaction occurs over the catalyst surface at $600{ }^{\circ} \mathrm{C}$. Steam provides the required heat of reaction, decreases rate of coke formation, and shifts thermodynamic equilibrium limitation toward completion. Feed to steam ratio is typically between 10 and 15 . The considered reactions in the model are:

$$
\begin{gathered}
\mathrm{C}_{8} \mathrm{H}_{10} \leftrightarrow \mathrm{C}_{8} \mathrm{H}_{8}+\mathrm{H}_{2} \\
\mathrm{C}_{8} \mathrm{H}_{10} \rightarrow \mathrm{C}_{6} \mathrm{H}_{6}+\mathrm{C}_{2} \mathrm{H}_{4} \\
\mathrm{C}_{8} \mathrm{H}_{10}+\mathrm{H}_{2} \rightarrow \mathrm{C}_{7} \mathrm{H}_{8}+\mathrm{CH}_{4} \\
\mathrm{C}_{8} \mathrm{H}_{8}+\mathrm{H}_{2} \rightarrow \mathrm{C}_{7} \mathrm{H}_{8}+\mathrm{CH}_{4} \\
\mathrm{H}_{2} \mathrm{O}+\mathrm{CH}_{4} \rightarrow \mathrm{CO}+3 \mathrm{H}_{2} \\
2 \mathrm{H}_{2} \mathrm{O}+\mathrm{C}_{2} \mathrm{H}_{4} \rightarrow \mathrm{CO}+4 \mathrm{H}_{2} \\
\mathrm{H}_{2} \mathrm{O}+\mathrm{CO} \rightarrow \mathrm{CO}_{2}+\mathrm{H}_{2}
\end{gathered}
$$

The main by-products in this process are benzene and toluene.The kinetic of thermal and catalytic reactions are adopted from related literatures [13-15].

Table 1 Catalyst and reactor specifications

\begin{tabular}{llll}
\hline & Bed 1 & Bed 2 & Bed 3 \\
\hline Feed & & & \\
\hline Temperature (K) & 886 & 898.2 & 897.6 \\
Flow rate & 8496.37 & From R-1 & From R-2 \\
Pressure (bar) & 1.25 & From R-1 & From R-2 \\
Steam rate & 7777 & & \\
Catalyst & & & \\
\hline Catalyst loading & 17 & 18 & \\
Diameter (m) & 0.0055 & & \\
Void fraction & 0.4312 & & \\
Density (kg m $\left.{ }^{-3}\right)$ & 2500 & & \\
\hline
\end{tabular}

\subsection{Auxiliary equations}

There are several parameters in the heterogeneous models such as heat and mass transfer coefficients between gas and solid phases that connects the gas and solid phases governing equations and they should be calculated from respecting correlations. Also, proper temperature and component dependent correlations should be applied to estimate thermal and physical properties of components and mixtures such as viscosity, specific heat capacity, heat conductivity and diffusion coefficients along the reactor. The references for considered correlations to calculate components and mixtures physical properties and mass and heat transfer coefficient between phases are summarized in Table 2.

Table 2 References for the considered correlations

\begin{tabular}{ll}
\hline Correlation & Reference \\
\hline Component heat capacity & {$[16]$} \\
Mixture heat capacity & - \\
Component viscosity (for $\mathrm{H}_{2}, \mathrm{H}_{2} \mathrm{O}$ ) & {$[17]$} \\
Component viscosity (for $\mathrm{C}_{8} \mathrm{H}_{10} \mathrm{C}_{8} \mathrm{H}_{8}, \mathrm{C}_{7} \mathrm{H}_{8}, \mathrm{C}_{6} \mathrm{H}_{6}$ ) & {$[17]$} \\
Binary mass diffusion coefficient i in j & {$[18]$} \\
Mixture viscosity & {$[18]$} \\
mass diffusion coefficient in mixture & {$[18]$} \\
Effectiveness factor & {$[19]$} \\
Mass transfer coefficient & {$[20]$} \\
Heat transfer coefficient & {$[21]$} \\
\hline
\end{tabular}

\section{Numerical solution}

The mass and energy governing equations create a set of nonlinear ordinary differential equations. This set of nonlinear differential equations can't be solved analytically and should be solved numerically. Runge-Kutta $4^{\text {th }}$ Order as an accurate and simple solution method has been selected to solve this set of equations.

\section{Integrated procedure for MOO}

In this study, the integrated procedure to create a multiobjective model (MOO) problem involves developing a multiobjective model, producing an optimal Pareto set and transferring developed multi objective model to a single objective optimization problem (SOO) to determine single solution point. The multi-objective model is formulated to maximize styrene production, and minimize side reactions via considering the feed temperature of reactors as decision variables. Then, the weighted sum method is used to transfer the developed multi objective problem to the single objective. The developed single optimal point of the composite function is determined by genetic algorithm. Genetic algorithm is a particular class of evolutionary algorithms in global optimization, which does not require gradient information [1]. It uses techniques inspired by evolutionary biology such as inherence, mutation, selection and crossover. In the genetic programming, a population with size of 100 and two basic operations, i.e., crossover probability of 0.9 and mutation probability of 0.1 are chosen. The considered multi-objective model in the proposed configuration can be formulated as follows: 
$\begin{array}{ll}\text { Maximize styrene production } & f_{1}(\vec{x}) \\ \text { Minimize toluene and benzene } & f_{2}(\vec{x})\end{array}$

$\vec{x}$ is decision variable vector, that contains the inlet temperature of first, second and third reactors. Due to thermodynamic equilibrium limitations, coke formation and hydrocarbon cracking, a temperature of $700{ }^{\circ} \mathrm{C}$ is chosen as the upper bound of inlet feed and temperature profile along the reactors. The bounds of decision variables are:

$$
T_{\mathrm{i}} \leq 700^{\circ} \mathrm{C} \quad i=1,2,3
$$

The considered objective functions are the main economic objectives in the styrene plant to increase production capacity and profit. Figure 2 shows a schematic depiction of considered optimization algorithm.

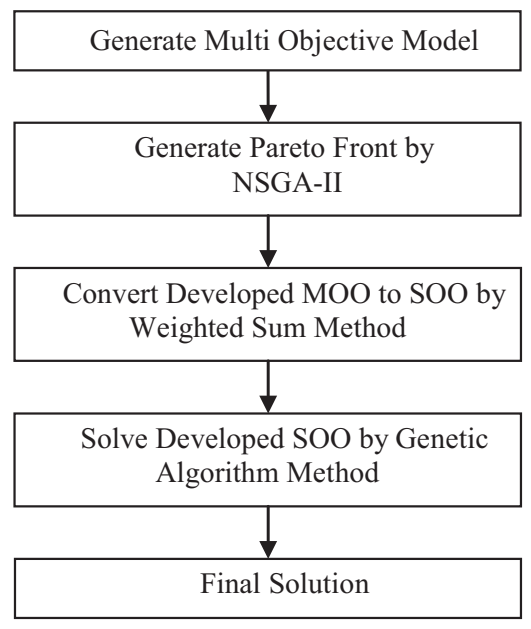

Fig. 2 Schematic of developed optimization algorithm

\section{Results and discussion}

In this section, the process performance to produce styrene is analyzed and the predicted component molar flow rate, temperature, and selectivity are presented. Simulation results are validated against the plant data to demonstrate the accuracy of the considered model. The comparison between simulation results and plant data is presented in Table 3. It is observed that the predicted styrene conversion and outlet temperature have a good agreement with the observed plant data.

Table 3 Comparison between simulation results and plant data

\begin{tabular}{llll}
\hline & Plant data & Simulation & R.E. (\%) \\
\hline Outlet Temperature & 850 & 848.2 & 0.2 \\
Conversion & 47.3 & 47.9 & 1.26 \\
\hline
\end{tabular}

Conventionally, styrene plant has large capacity. Hence, the investment and operational cost of that plant is high, so that any small enhancement in the process and production capacity can yield significant financial rewards. In this section, the simulation results of the conventional axial flow and optimized spherical configurations are presented at steady state condition. Figure 3 indicates the Pareto front for the considered multiobjective model. It is possible to visualize Pareto frontier in two spaces, namely two considered objectives, $\mathrm{f}_{1}$ and $\mathrm{f}_{2}$.

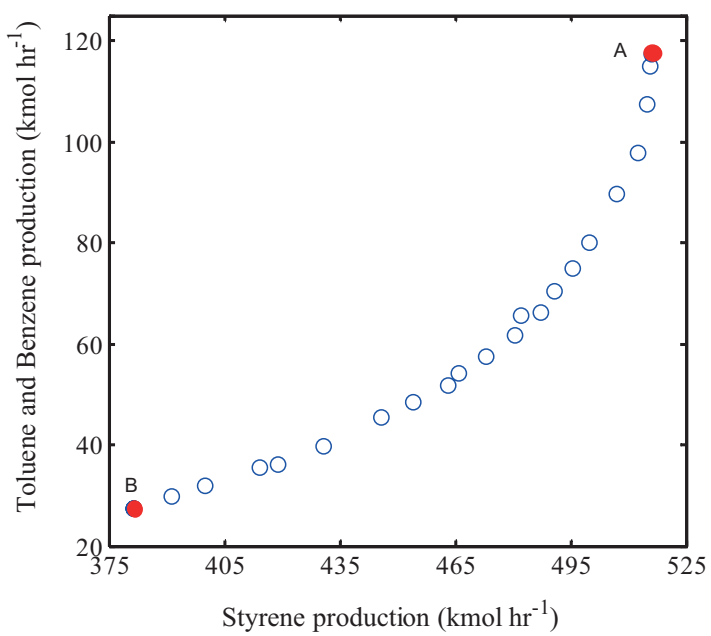

Fig. 3 Pareto front of considered multi objectives

It seems that a considerable enhancement in the styrene production from 381.5 to $516.3 \mathrm{kmol} \mathrm{h}^{-1}$ leads to a drastic increment of by-product from 27.4 to $117.2 \mathrm{kmol} \mathrm{h}^{-1} \mathrm{kmol} \mathrm{h}^{-1}$. According to Fig. 2, the maximum styrene and by-product capacities occur simultaneously. If the styrene production be considered as the single objective function, the design point $\mathrm{A}$ is the optimal design point, while the design point $\mathrm{B}$ indicates the optimum system performance considering minimum by-product formation as the single objective function. In this section, the MOO model is converted to a SOO problem using weighted sum method and the single optimum point is found by genetic algorithm:

$$
\mathrm{f}(\mathrm{x})=-\omega_{1} \mathrm{f}_{\text {Styrene }}+\omega_{2}\left(\mathrm{f}_{\text {Toluene }}+\mathrm{f}_{\text {Benzene }}\right)
$$

The selected weights for objectives $\mathrm{f}_{1}$ and $\mathrm{f}_{2}$ are 0.8 and 0.2 , respectively. Table 4 represents the obtained values for decision variables and results of the optimized processes. The presented results show that the obtained optimal feed temperature to each reactor is feasible and can be created by inter-stage heaters. There is a dramatic difference between calculated optimal temperature in the spherical configuration and conventional condition.

Table 4 Optimal condition of spherical configuration

\begin{tabular}{llll}
\hline & \multicolumn{3}{c}{ Optimized spherical } \\
\hline & $1^{\text {st }}$ & $2^{\text {st }}$ & $3^{\text {st }}$ \\
\hline Feed Temperature $(\mathrm{K})$ & 870.9 & 885.5 & 910.8 \\
Conversion & 82.7 & & \\
Styrene Production & 504.2 & & \\
\hline
\end{tabular}


Although third spherical reactor experiences the higher temperature compared to the conventional reactor, first and second spherical reactors experience a lower feed temperature. In this section, conversion and productivity as the main parameters to investigate the process performance are compared for two configurations. Figure 4 shows ethylbenzene conversion as the main reactant to produce styrene, along the reactors at steady state condition. The ethylbenzene is converted to the styrene through catalytic and thermal dehydrogenation, cracking, and hydrogenation reactions. It decreases from 707 to $121.6 \mathrm{kmol} \mathrm{h}^{-1}$ in the spherical process. Ethylbenzene conversion in the first, second and third spherical reactors are about $28.8 \%, 37.5 \%$ and $61.3 \%$, respectively.

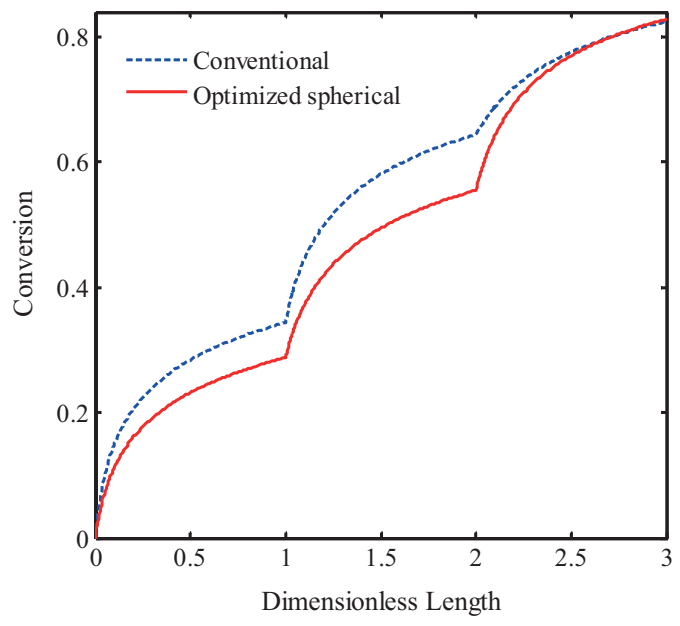

Fig. 4 Ethylbenzene conversion along the conventional and optimized processes

The overall ethylbenzene conversion in the conventional and optimized spherical configurations is about $82.2 \%$ and $82.8 \%$, respectively. In the optimal condition of spherical configuration, the ethylbenzene conversion has been improved about $0.6 \%$ compared to the conventional process. Ethylbenzene is converted to styrene as the main product, benzene, toluene, ethylene and methane as by-products. Selectivity as main parameter to investigate side reactions, which is defined as ratio of produced styrene per consumed ethylbenzene, in the conventional and optimized configurations is about is about $85 \%$ and $85.7 \%$, respectively. Selectivity in the proposed configuration is improved about $0.86 \%$ compared to the conventional process. Lower conversion in the first and second spherical reactors has been compensated the applying higher feed temperature in the third reactor. Figure 5 shows the flow rate of styrene along the conventional and optimized spherical configurations. According to this figure, produced styrene in the first, second, and third optimized spherical reactors are about $244.5,177$ and $74.7 \mathrm{kmol} \mathrm{h}^{-1}$. The produced styrene in the conventional and optimized spherical configurations is about 497.3 and $504.2 \mathrm{kmol} \mathrm{h}^{-1}$. Styrene production in the proposed configuration has been enhanced about $1.39 \%$ compared to the conventional process. In other words, styrene capacity is improved about $60000 \mathrm{kmol}$ per year at optimized condition. The higher reactant concentration in the first reactor causes the higher styrene production and selectivity. Styrene production in the third reactor has approached to the equilibrium condition because of lower reactant concentration over the catalyst and higher feed temperature. Increasing styrene concentration along the reactors increases rate of styrene to toluene dehydrogenation and decrease selectivity in the reaction zone.

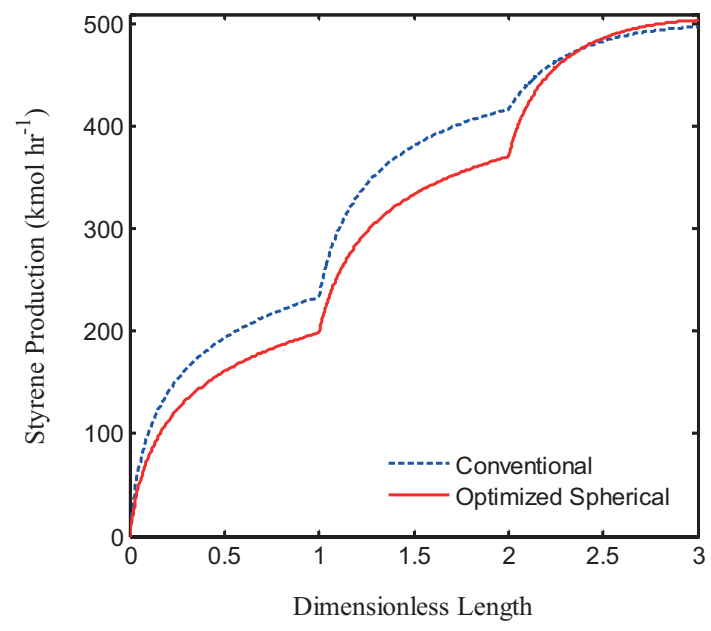

Fig. 5 Styrene flow rates along reactors

Figure 6 shows the pressure profile along the reactors. In the chemical process, pressure drop is a serious problem that limits the production capacity and increases operational cost. The radial flow spherical reactors have lower pressure drop compared to the conventional axial flow reactors. Requiring lower thickness material is other main advantages of spherical reactors compared to tubular reactors. In addition, smaller catalytic pellets with higher effectiveness factor and higher molar flow rate of the feed can be applied in the radial flow reactors due to lower pressure drop. As it can be seen in Fig. 4, pressure drop in the spherical reactor is about 0.16 , while it is about 0.5 atm in the industrial tabular reactor. Comparison between pressure drop in the spherical configuration and the conventional tubular reactor shows that the pressure drop in the conventional process is three times larger than spherical configuration. The flow in the spherical reactors is radial so it offers a large mean crosssectional area and small distance for the flow path compared to the traditional tabular reactors. Decreasing pressure drop is one of the main advantages of proposed spherical configuration that reduces operational costs and permits higher feed flow rate.

Figure 7 (a-c) shows the rate of toluene, methane and benzene along the reactors. According to this figure, outlet toluene and methane from third reactor are about 73.6 and 57.83 $\mathrm{kmol} \mathrm{h}^{-1}$, respectively. The methane and toluene are produced through styrene and ethylbenzene hydrogenation reactions. A part of methane is converted to hydrogen through thermal steam-reforming. The produced benzene in the conventional 
and optimized process is about 13.3 and $12.7 \mathrm{kmol} \mathrm{h}^{-1}$, respectively. Toluene and benzene production have been decreases about $2.2 \%$ and $4.7 \%$ in the optimized configuration.

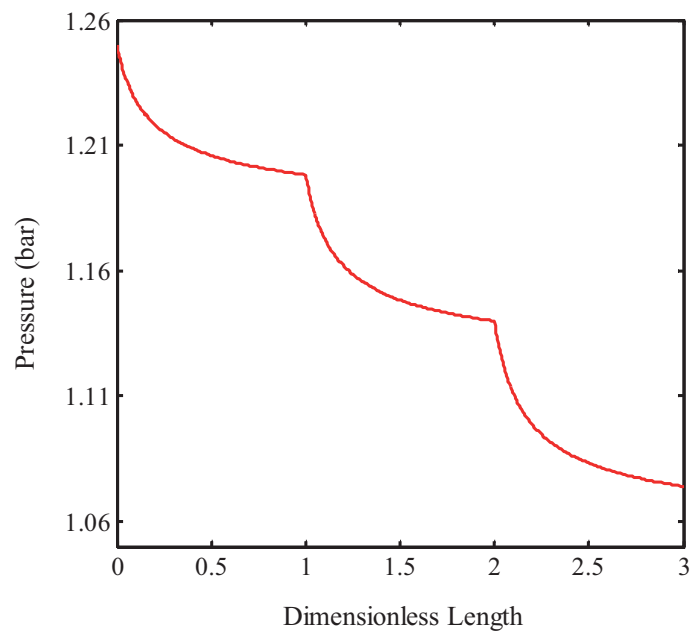

Fig. 6 Pressure profile along the reactors

In other word, production capacity of toluene and benzene are decreased about 18000 and $5000 \mathrm{kmol}$ per year at the optimized condition. The benzene and ethylene are produced through ethylbenzene cracking reaction. Steam reforming of ethylene decreases ethylene concentration in the reactors and shifts ethylbenzene cracking reaction toward the right side and results in higher benzene production. Generally, styrene and ethylbenzene hydrogenation and ethylbenzene cracking reactions decrease selectivity of process in the styrene plant. It is appeared that, applying higher feed temperature in the third reactor enhances ethylbenzene cracking and hydrogenation reactions and increases toluene and benzene production. This figure shows that the feedstock temperature has an effective role on the by-product formation.

Figure 8 shows the temperature profile along the conventional and optimized spherical reactors. In this process temperature decreases due to endothermic reactions. Although the maximum reactant concentration is appeared in the first reactor, the maximum temperature drop is experienced in the second reactor due to higher feed temperature compared to the first reactor. Temperature drop drastically decreases the rate of thermal cracking, dehydrogenation and hydrogenation reactions and has a moderate effect on the catalytic rates. As can be seen, although the lower temperature in the first spherical reactor decreases ethylbenzene conversion, it increases styrene selectivity as one of the main objects. The higher temperature in the third reactor causes higher ethylbenzene conversion and by-product formation and also decreases process selectivity in the styrene plant. In addition, the results show that temperature profile in the third reactor approaches to the equilibrium condition due to decreasing ethylbenzene concentration over the catalyst. Since rate of reactions is highly temperature dependent, particularly in the thermal reactions, lower feed temperature in the first and second reactors decreases rate of styrene production at the entrance of reactors.
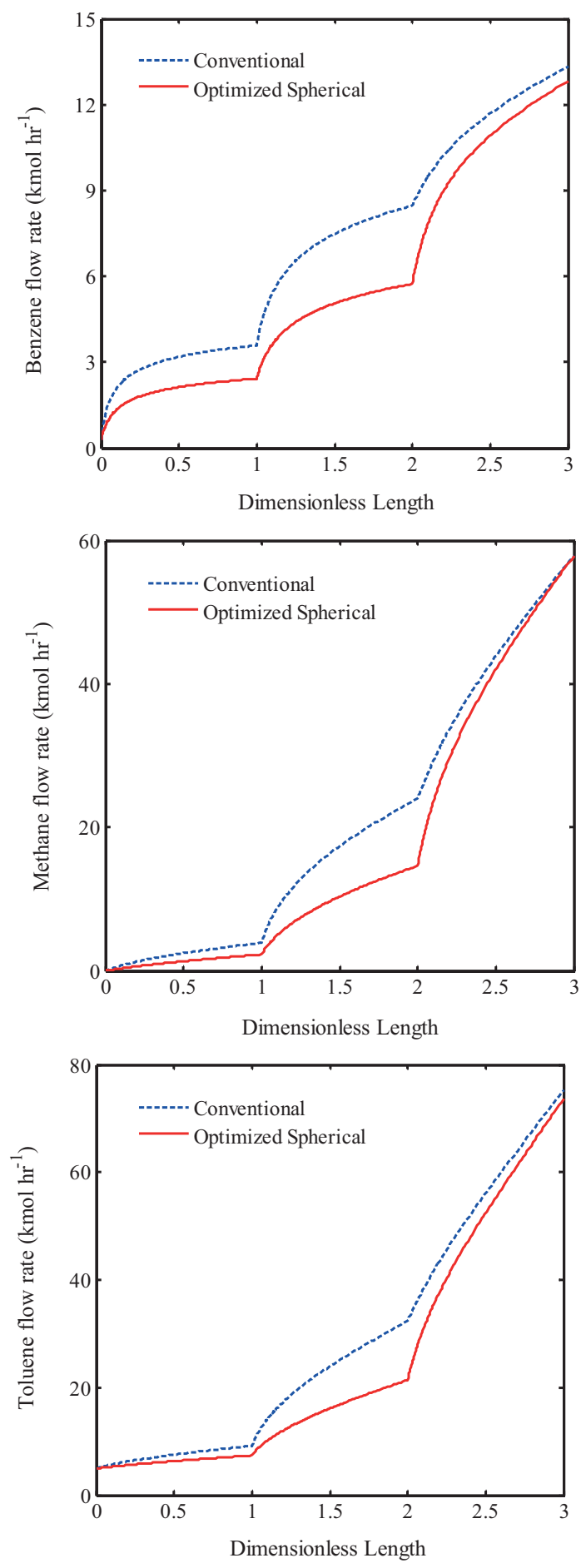

Fig. 7 (a-c) Benzene, methane and toluene flow rates along the reactors 


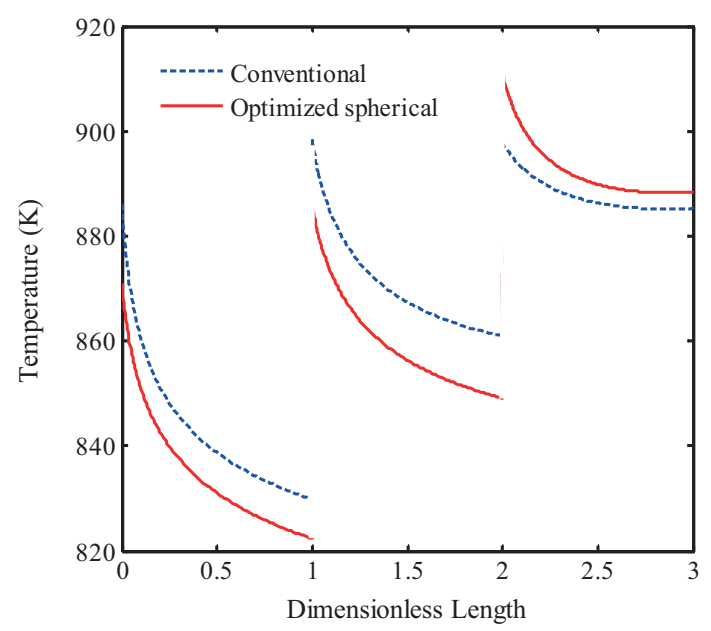

Fig. 8 Temperature profile along the reactors

\section{Conclusion}

In this study ethylbenzene dehydrogenation in the radial flow spherical reactors were modelled and optimized based on the mass and energy conservation laws at steady state condition. To present an exact mathematical model, as well as styrene dehydrogenation reaction, catalytic and thermal side reactions such as ethylbenzene hydrogenation, cracking, and styrene hydrogenation reactions were considered in the developed model. In the optimization stage the optimal feed temperature of reactors was calculated to maximize styrene production and minimize toluene and benzene production. the developed multi-objective model was converted to a single objective problem by the weighted sum method, to achieve a single optimal point. Then, the single optimum point was found by genetic algorithm. The simulation results showed that styrene production in the optimized spherical configuration is improved $1.39 \%$ compared to the conventional configuration. In addition, predicted styrene selectivity and ethylbenzene conversion were improved about $0.86 \%$ and $0.61 \%$ at the optimized condition, respectively. The pressure drop in the spherical and conventional configurations is 0.16 and 0.5 bar that proved the efficiency of the spherical configuration to produce styrene.

\section{Nomenclature}

$A_{c} \quad$ Cross section area $\left(\mathrm{m}^{2}\right)$

$a_{v} \quad$ Specific surface area of catalyst pellet $\left(\mathrm{m}^{2} \mathrm{~m}^{-3}\right)$

$\mathrm{C}_{\mathrm{i}} \quad$ Molar concentration of component $\mathrm{i}\left(\mathrm{mol} \mathrm{m}^{-3}\right)$

$\mathrm{C}_{\mathrm{p}} \quad$ Specific heat capacity $\left(\mathrm{J} \mathrm{mol}^{-1} \mathrm{~K}^{-1}\right)$

D Diameter (m)

$D_{i j} \quad$ Binary diffusion coefficient of $i$ in $j\left(\mathrm{~m}^{2} \mathrm{~s}^{-1}\right)$

$\mathrm{D}_{\mathrm{im}}$ Diffusion coefficient of $\mathrm{i}$ in mixture $\left(\mathrm{m}^{2} \mathrm{~s}^{-1}\right)$

f Friction factor

$\mathrm{h}_{\mathrm{f}} \quad$ Gas-solid heat transfer coefficient $\left(\mathrm{W} \mathrm{m}^{-2} \mathrm{~K}^{-1}\right)$

$\mathrm{K}_{\mathrm{eq}} \quad$ Equilibrium constant $\left(\mathrm{m}^{3} \mathrm{~mol}^{-1}\right)$

$\mathrm{K}_{\mathrm{i}} \quad$ Adsorption equilibrium constant for $\mathrm{i}\left(\mathrm{m}^{3} \mathrm{~mol}^{-1}\right)$ $\mathrm{k}_{\mathrm{g}} \quad$ Mass transfer coefficient $\left(\mathrm{m} \mathrm{s}^{-1}\right)$

$\mathrm{k}_{\mathrm{i}} \quad$ rate constant for $\mathrm{i}^{\text {th }}$ catalytic reaction $\left(\mathrm{r}_{\mathrm{ci}}\right)$

$\mathrm{k}_{\mathrm{ti}} \quad$ rate constant for $\mathrm{i}^{\text {th }}$ thermal reaction $\left(\mathrm{r}_{\mathrm{ti}}\right)$

P Total pressure (bar)

$\mathrm{P}_{\mathrm{i}} \quad$ Component partial temperature (bar)

$\mathrm{r} \quad$ Radial reactor coordinate $(\mathrm{m})$

$r_{c i} \quad$ Rate of $i^{\text {th }}$ catalytic reaction $\left(\mathrm{mol} \mathrm{kg}^{-1} \mathrm{~s}^{-1}\right)$

$r_{t i} \quad$ Rate of $i^{\text {th }}$ thermal reaction $\left(\mathrm{mol} \mathrm{m}^{-3} \mathrm{~s}^{-1}\right)$

$\mathrm{T}$ Temperature (C)

$\mathrm{u}_{\mathrm{g}} \quad$ Linear velocity of fluid phase $\left(\mathrm{m} \mathrm{s}^{-1}\right)$

\section{Greek letters}

$\rho \quad$ Density of fluid phase $\left(\mathrm{kg} \mathrm{m}^{-3}\right)$

$\eta \quad$ Effectiveness factor

$\Delta \mathrm{H} \quad$ Heat of reaction $\left(\mathrm{J} \mathrm{mol}^{-3}\right)$

$\Delta \mathrm{P} \quad$ Pressure difference $(\mathrm{Pa})$

\section{Superscripts}

$\mathrm{G}$ In the bulk gas phase

P Particle

S At the catalyst surface

\section{References}

[1] Quicker, H. C., Dittmeyer, P. R. "Mathematical simulation of catalytic dehydrogenation of ethylbenzene to styrene in a composite palladium membrane reactor." Journal of Membrane Science. 136(1-2), pp. 161 172. 1997. DOI: 10.1016/s0376-7388(97)81990-4

[2] Castor, J. D. W. "Ullmann's Encyclopedia of Industrial Chemistry." Wiley Online Library, New York, 1994.

[3] Abdalla, B., Elnashaie, S., Alkhowaiter, S., Elshishini, S. "Intrinsic kinetics and industrial reactors modelling for the dehydrogenation of ethylbenzene to styrene on promoted iron oxide catalysts." Applied Catalysis A: General. 113(1), pp. 89-102. 1994.

DOI: $10.1016 / 0926-860 x(94) 80243-2$

[4] Clough, D. E., Ramirez, W. F. "Mathematical modeling and optimization of the dehydrogenation of ethylbenzene to form styrene." AIChE Journal. 22(6), pp. 1097-1105. 1976. DOI: 10.1002/aic.690220621

[5] Tamsilian, Y., Ebrahimi, A. N., Ahmad Ramazani, S. A., Abdollahzadeh, H. "Modeling and sensitivity analysis of styrene monomer production process and investigation of catalyst behavior." Computers \& Chemical Engineering. 40, pp. 1-11. 2012.

DOI: 10.1016/j.compchemeng.2012.01.014

[6] Mousavi, S. M., Panahi, P. N., Niaei, A., Farzi, A., Salari, D. "Modeling and Simulation of Styrene Monomer Reactor: Mathematical and Artificial Neural Network Model." International Journal of Scientific \& Engineering Research. 3(3), pp. 1-7. 2012.

[7] Elnashaie, S. S., Abdalla, B. K., Hughes, R. "Simulation of the industrial fixed bed catalytic reactor for the dehydrogenation of ethylbenzene to styrene: heterogeneous dusty gas model." Industrial \& Engineering Chemistry Research. 32(11), pp. 2537-2541. 1993.

DOI: $10.1021 / \mathrm{ie} 00023 \mathrm{a} 016$

[8] Abo-Ghander, N. S., Grace, J. R., Elnashaie, S. S. E. H., Lim, C. J. "Modeling of a novel membrane reactor to integrate dehydrogenation of ethylbenzene to styrene with hydrogenation of nitrobenzene to aniline." Chemical Engineering Science. 63(7), pp. 1817-1826. 2008. DOI: 10.1016/j.ces.2007.12.007 
[9] Abo-Ghander, N. S., Logist, F., Grace, J. R., Van Impe, J. F. M., Elnashaie, S. S. E. H., Lim, C. J. "Optimal design of an autothermal membrane reactor coupling the dehydrogenation of ethylbenzene to styrene with the hydrogenation of nitrobenzene to aniline." Chemical Engineering Science. 65(10), pp. 3113-3127. 2010. DOI: 10.1016/j.ces.2010.02.007

[10] Farsi, M., Rokhgireh, A., Javidi, M. "Modelling and optimization of simultaneous styrene and hydrogen production in an industrial hydrogenpermselective membrane reactor." The Canadian Journal of Chemical Engineering. 93(1), pp. 46-54. 2015. DOI: 10.1002/cjce.22103

[11] Burke, E. K., Kendall, G. "Search methodologies." Springer, Heidelberg, 2005. DOI: $10.1007 / 0-387-28356-0$

[12] Bhaskar, V., Gupta, S., Ray, A. "Applications of multi-objective optimization in chemical engineering." Reviews in Chemical Engineering. 16, pp. 1-54. 2000. DOI: 10.1515/revce.2000.16.1.1

[13] Devoldere, K. R., Froment, G. F. "Coke formation and gasification in the catalytic dehydrogenation of ethylbenzene." Industrial \& Engineering Chemistry Research. 38(7), pp. 2626-2633. 1999. DOI: 10.1021/ie980169+

[14] Marler, R. T., Arora, J. S. "Survey of multi-objective optimization methods for engineering." Structural and Multidisciplinary Optimization. 26(6), pp. 369-395. 2004. DOI: 10.1007/s00158-003-0368-6
[15] Sundaram, K., Froment, G. "Modeling of thermal cracking kinetics-II: Cracking of iso-butane, of n-butane and of mixtures ethane-propanen-butane." Chemical Engineering Science. 32(6), pp. 609-617. 1977. DOI: 10.1016/0009-2509(77)80226-1

[16] Reid, R. C., Prausnitz, J. M., Poling, B. E. "The properties of gases and liquids." McGraw-Hill, New York, 1987.

[17] Sherwood, T. K., Reid, R., Prausnitz, J. "The properties of gases and liquids." McGraw-Hill, New York, 1977.

[18] Aguilar, R., Poznyak, A., Martínez-Guerra, R., Maya-Yescas, R. "Temperature control in catalytic cracking reactors via a robust PID controller." Journal of Process Control. 12(6), pp. 695-705. 2012.

DOI: 10.1016/s0959-1524(01)00034-8

[19] Lee, W. J., Froment, G. F. "Ethylbenzene dehydrogenation into styrene: kinetic modeling and reactor simulation." Industrial \& Engineering Chemistry Research. 47(23), pp. 9183-9194. 2008. DOI: 10.1021/ie071098u

[20] Cussler, E. L. "Diffusion: mass transfer in fluid systems." Cambridge University Press, New York, 2009.

[21] Holman, J. "Heat Transfer, SI Metric Edition." McGraw-Hill, New York, 1989. 